\title{
Tolerância à dessecação e armazenamento de sementes de Myrcianthes pungens (O. Berg) D. Legrand (Myrtaceae)
}

\author{
iD Marina Crestana Guardia ${ }^{1}$, (D) Lilian Maria Asperti ${ }^{1}$, Giovanna de Macedo Cancian ${ }^{1}$ e (D) Claudio José Barbedo \\ Recebido: 17.03.2020; aceito: 09.07.2020
}

Como citar: Guardia, M.C., Asperti, L.M., Cancian, G.M., \& Barbedo, C.J. 2020. Tolerância à dessecação e armazenamento de sementes de Myrcianthes pungens (O. Berg) D. Legrand (Myrtaceae). Hoehnea 47: e192020. http://dx.doi.org/10.1590/2236-8906-19/2020.

ABSTRACT - (Desiccation tolerance and storage of seeds of Myrcianthes pungens (O. Berg) D. Legrand (Myrtaceae)). Many barriers are found in the production of plantlets of species native to the tropics, largely due to the lack of knowledge about their seeds. Among these, the lack of technology to long store of recalcitrant seeds can also be a great risk to the loss of important plant diversity. In this work we analyzed the desiccation tolerance and the storage capacity of recalcitrant seeds of Myrcianthes pungens (O. Berg) D. Legrand. The results showed that $M$. pungens seeds are intolerant to desiccation up to $30 \%$ water, are better stored under low temperatures and, under high temperatures, have high rates of respiration and other oxidative processes.

Keywords: Myrtaceae, recalcitrance, seed respiration

RESUMO - (Tolerância à dessecação e armazenamento de sementes de Myrcianthes pungens $(\mathrm{O}$. Berg) D. Legrand (Myrtaceae)). Muitas barreiras são encontradas na produção de plântulas de espécies nativas dos trópicos, em grande parte devido ao desconhecimento de suas sementes. Entre elas, a falta de tecnologia para armazenamento prolongado de sementes recalcitrantes, que também pode ser um grande risco para a perda de importante diversidade vegetal. Neste trabalho, analisamos a tolerância à dessecação e a capacidade de armazenamento de sementes recalcitrantes de Myrcianthes pungens (O. Berg) D. Legrand. Os resultados mostraram que as sementes de M. pungens são intolerantes à dessecação de até $30 \%$ de água, são melhor armazenadas em baixas temperaturas e, sob altas temperaturas apresentam altas taxas de respiração e outros processos oxidativos.

Palavras-chave: Myrtaceae, recalcitrância, respiração de sementes

\section{Introdução}

A conservação da viabilidade durante $\mathrm{o}$ armazenamento das sementes, principalmente com a descoberta de um número cada vez maior de sementes intolerantes à dessecação, denominadas recalcitrantes, tem sido um dos aspectos mais visados nos últimos anos (Linkies et al. 2010, Marcos Filho 2015, Barbedo 2018, Barbedo \& Santos Junior 2018, Magnani 2018, Silva et al. 2018, Lamont et al. 2019 , Subbiah et al. 2019, Wu et al. 2019).
A falta de tecnologia que permita armazenar essas sementes por longos períodos, como os exigidos em bancos de germoplasma, tem colocado em risco a própria sobrevivência das espécies (Barbedo et al. 2018). Uma das maiores dificuldades no desenvolvimento dessas tecnologias reside na própria falta de conhecimento sobre a fisiologia das sementes recalcitrantes, uma vez que os comportamentos são variáveis até mesmo dentro da própria espécie, quando se variam as condições nas quais as sementes foram produzidas.

1. Instituto de Botânica, Núcleo de Pesquisa em Sementes, Avenida Miguel Estéfno, 3.687, 04301-902 São Paulo, SP

2. Autor para correspondência: cjbarbedo@yahoo.com.br 
Espécies frutíferas nativas do Brasil vêm despertando interesse econômico cada vez maior face à crescente diversidade de usos descobertos para cada parte da planta (Lamarca et al. 2013a, Silva \& Lamarca 2018). Contudo, grandes entraves no manejo de essências nativas são encontrados na produção de suas mudas e, em grande parte, devido à falta do conhecimento sobre suas sementes. Dentre essas espécies figura o guabiju, Myrcianthes pungens (O. Berg) D. Legrand (Myrtaceae).

Myrcianthes pungens é uma espécie semidecídua, climácica, esciófita, de ocorrência tanto no Cerrado quanto na Mata Atlântica, geralmente em solos úmidos e rochosos. Produz frutos suculentos, de cor roxo-escura e sabor agradável, pouco explorados comercialmente, apesar de possuírem elevados teores de antocianina e carotenoides, bem como elevada atividade antioxidante (Andrade $e t$ al. 2011, Nora et al. 2014). Pelas suas características de grande longevidade, potencial apícola e frutos comestíveis, é indicada sua utilização em plantios mistos de conservação e restauração de áreas degradadas.

A despeito de não terem sido realizados trabalhos específicos sobre tolerância à dessecação nessa espécie, que permitissem identificar o menor teor de água tolerado, as sementes de $M$. pungens são consideradas sensíveis à dessecação (recalcitrantes), fotoblásticas positivas e, dependendo das condições ambientais, podem ser armazenadas por até 10 meses (Santos et al. 2004, Fior et al. 2010, Gibbert et al. 2019). Evidentemente este período é insuficiente para sua inserção em bancos de germoplasma, necessitando estudos que procurem compreender o processo de deterioração dessas sementes, bem como sua tolerância à dessecação. Neste trabalho procurou-se avaliar a tolerância à dessecação e a capacidade de armazenamento de sementes recalcitrantes de Myrcianthes pungens.

\section{Material e métodos}

Material vegetal - Frutos de Myrcianthes pungens (O.Berg) D.Legrand foram obtidos de duas matrizes plantadas e mantidas em condições de campo, em propriedade particular, na cidade de São Paulo, SP, Brasil (2327'15”S, 46 44'45”O, altitude $832 \mathrm{~m}$ ) e levados ao Laboratório do Núcleo de

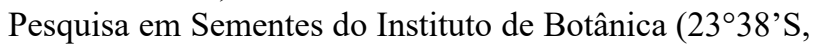
$46^{\circ} 37^{\circ} \mathrm{O}, 785 \mathrm{~m}$ ), na mesma cidade.

As coletas ocorreram após o início de queda espontânea dos frutos, que apresentavam aspecto típico da maturação com coloração roxo-escura, entre os meses de janeiro e fevereiro, nos quais a temperatura média foi de $25^{\circ} \mathrm{C}$ e a precipitação média de $125 \mathrm{~mm}$.

No laboratório, as sementes foram extraídas manualmente, beneficiadas (removendo-se as danificadas ou fora do padrão do lote) e armazenadas em sacos de polietileno transparentes com $2 \mathrm{~mm}$ de espessura (SPT), a $10^{\circ} \mathrm{C}$, até a instalação dos experimentos, não ultrapassando 15 dias. Imediatamente após a extração e o beneficiamento, as sementes foram analisadas quanto a teor e potencial de água e germinação, conforme descrito a seguir.

Análises físicas e fisiológicas - O teor de água foi determinado gravimetricamente, pelo método de estufa a $105{ }^{\circ} \mathrm{C}$ por 24 horas, com cinco sementes por repetição, e os resultados apresentados em porcentagem, em base úmida (Brasil 2009). O potencial de água dos embriões (5 sementes por repetição) foi medido em potenciômetro WP4 (Decagon Devices, Pullman, EUA), baseando-se na temperatura do ponto de orvalho do ar em equilíbrio com a amostra examinada (Bonjovani \& Barbedo 2014).

$O$ teste de germinação (15 sementes por repetição) foi conduzido em rolos de papel Germitest, previamente umedecidos (Brasil 2009), mantidos em câmaras de germinação com $100 \%$ de umidade relativa do ar, reguladas para $25 \pm 1^{\circ} \mathrm{C}$, com luz contínua (Santos et al. 2004). As avaliações foram realizadas a cada três dias durante 60 dias, considerando-se como germinadas as sementes com emissão de raiz primária de, no mínimo, $5 \mathrm{~mm}$, sendo os resultados apresentados em porcentagem. Como medida de vigor das sementes foram avaliados, ainda, o tempo médio de germinação (TMG), a variância do tempo médio de germinação (VTM) e o índice de velocidade de germinação (IVG), segundo fórmulas descritas em Borghetti \& Ferreira (2004) e Santana \& Ranal (2004). Foram também computadas as sementes que produziram plântulas normais, ou seja, que apresentaram sistema radical e parte aérea bem desenvolvidos e sem defeitos aparentes, com resultados apresentados em porcentagem.

Taxas de respiração das sementes - as taxas respiratórias e a presença de outras reações oxidativas nas sementes foram obtidas por meio da avaliação do consumo de oxigênio $\left(\mathrm{O}_{2}\right)$ e da produção de dióxido de carbono $\left(\mathrm{CO}_{2}\right)$. As sementes foram incubadas em respirômetros (frascos herméticos, perfurados e selados para tomada de amostras de ar), segundo metodologia descrita por Lamarca \& Barbedo (2012).

Antes da introdução nos respirômetros, a massa seca e o volume das sementes foram determinados, bem como o volume de ar do respirômetro, seguindo metodologia descrita em Lamarca \& Barbedo (2012). O fechamento deste foi considerado o tempo zero, correspondendo à atmosfera normal ( $21 \%$ de oxigênio e $0,03 \%$ de dióxido de carbono). Após 15 dias de incubação, amostras de ar foram tomadas e analisadas em analisador Modelo 6600 (Illinois Instruments, Inc., Johnsburg, EUA), baseando-se em sensor potenciométrico, com eletrólito sólido cerâmico na forma de tubo à base de óxido de zircônio $\left(\mathrm{ZrO}_{2}\right)$ para o $\mathrm{O}_{2} \mathrm{e}$, em sensor de infravermelho para $\mathrm{CO}_{2}$ (Lamarca \& Barbedo 2012). Os valores, em porcentagem de volume do gás analisado, foram convertidos para pressão parcial do gás, considerando-se a pressão atmosférica local como 0,9 atm, e para $\mu \mathrm{mol} \mathrm{de} \mathrm{O}_{2}$ e de $\mathrm{CO}_{2}$, pela equação de Clapeyron. Os 
valores assim obtidos foram divididos pela massa seca (MS) das sementes colocadas nos respirômetros e pelo número de dias em que permaneceram incubadas, obtendo-se o valor expresso em $\mu \mathrm{mol}$ por grama de MS por dia $(\mu \mathrm{mol}$ $\left.\mathrm{gMS}^{-1} \mathrm{~d}^{-1}\right)$. Foi calculado, também, o quociente respiratório $(\mathrm{QR})$, dividindo-se o valor obtido para produção de $\mathrm{CO}_{2}$ pelo obtido para consumo de $\mathrm{O}_{2}\left(\mathrm{QR}=\mathrm{CO}_{2} \mathrm{O}_{2}^{-1}\right)$, ambos em $\mu \mathrm{mol} \mathrm{gMS}^{-1} \mathrm{~d}^{-1}$ (Kader \& Saltveit 2002).

Secagem e armazenamento das sementes - as sementes foram submetidas a secagem intermitente para reduzir seu teor de água, inicialmente de $50 \%$, até valores próximos a $40 \%$ e $30 \%$, denominados níveis de secagem. Intercalaramse períodos de 12 horas em estufa com circulação forçada de ar, regulada para $40 \pm 2{ }^{\circ} \mathrm{C}$, com períodos de 12 horas de repouso, em SPT a $10{ }^{\circ} \mathrm{C}$. Uma amostra de cada lote foi utilizada para controle da perda de peso e cálculo do teor de água provável do lote ao longo da secagem. Os tempos necessários para atingir valores aproximados àqueles teores de água foram, respectivamente, um e quatro dias. Estabeleceram-se, desta forma, três níveis para o fator teor de água: $50 \%, 40 \%$ e $30 \%$. Amostras de sementes de cada nível foram armazenadas em SPT, em câmara fria $\left(10^{\circ} \mathrm{C}\right)$ e em condição natural de laboratório $\left(25^{\circ} \mathrm{C}\right)$, sob luz ou escuro contínuos. Essas sementes foram avaliadas quanto à sua qualidade fisiológica imediatamente após a secagem e aos 15, 30, 66, 180 e 240 dias de armazenamento. Os testes realizados com 180 e 240 dias foram com sementes com teor de água de $50 \%$ armazenadas à $10^{\circ} \mathrm{C}$.

Delineamento experimental e análise estatística - o delineamento experimental utilizado foi o inteiramente casualizado, com quatro repetições, em esquema fatorial $3 \times$ 9 (teor de água $\times$ condição e período de armazenamento), exceto para sementes armazenadas por 180 e 240 dias. Os resultados obtidos foram analisados pelo teste $\mathrm{F}$ e as médias foram comparadas entre si pelo teste de Tukey, ao nível de 5\% (Santana \& Ranal 2004).

\section{Resultados e Discussão}

O teor de água das sementes no momento da colheita ( $c a$. $50 \%$ ) foi menor que os $68 \%$ obtidos por Santos et al. (2004), para a mesma espécie, e maior que os $41-44 \%$ obtidos por Fior et al. (2010). Segundo Barbedo et al. (2013) e Guardia et al. (2018), diferenças no teor de água no momento da dispersão e/ou colheita das sementes de uma mesma espécie podem indicar seu grau de maturidade, que será tanto maior quanto menor o teor de água. Os valores do teor de água situariam a maturidade das sementes deste trabalho entre os de Santos et al. (2004) e Fior et al. (2010). É importante salientar que nestes trabalhos os frutos foram colhidos em seu grau máximo de maturação. Portanto, assim como observado em sementes recalcitrantes de outras espécies, o grau máximo de maturação dos frutos não necessariamente está associado com o máximo de maturação das sementes
(Lamarca et al. 2013b, Barbedo 2018), uma vez que estas podem não ter atingido o máximo em características como acúmulo de massa seca, capacidade de germinação e vigor das sementes (Guardia et al. 2018).

O conteúdo de massa seca das sementes foi de $275 \mathrm{mg}$ semente $^{-1}$, que por sua vez, foi superior ao descrito para a espécie em trabalhos anteriores. O conteúdo de massa seca aumenta com o avanço da maturação das sementes (Marcos Filho 2015), portanto, os maiores valores de massa seca das sementes obtidos em relação aos de Santos et al. (2004) eram esperados, uma vez que as sementes apresentavam teor de água mais elevado. Contudo, o fato de serem mais elevados inclusive que os de Fior et al. (2010), provavelmente não se deve à diferença na maturidade, mas às condições nas quais as sementes foram formadas, como já verificado para outras espécies (Borges et al. 2005, Lamarca et al. 2016). Variações intrapopulacionais e fatores de sazonalidade podem, de fato, influenciar os resultados, como observado em sementes de baru (Dipteryx alata Vog.), por exemplo, em que frutos e sementes apresentaram variação anual quanto às suas características morfológicas. Estes autores observaram que, dividindo as plantas em grupos, menos da metade das plantas permaneceu no mesmo grupo durante dois anos (Sano et al. 1999).

Os teores de água obtidos após as secagens, $43 \%$ e $30 \%$, foram muito próximos aos definidos inicialmente $(40 \% \mathrm{e}$ $30 \%)$. Já os potenciais de água indicaram que a secagem não apenas reduziu a quantidade de água, mas modificou o tipo e suas propriedades termodinâmicas. O potencial de água das sementes de $M$. pungens não submetidas à secagem, que foi de -1,04 MPa, caracterizou a presença de água do tipo 5 (> -1,5 MPa, segundo Vertucci 1993), ou seja, água livre. Nesse nível de hidratação há água livre suficiente, por exemplo, para que se inicie a germinação. Quando as sementes foram secas até $43 \%$, o potencial de água, mensurado em $-12,5$ $\mathrm{MPa}$, indicou a mudança para água do tipo 3 (entre -4 e -11 MPa) que, em sementes recalcitrantes, frequentemente acelera os processos de deterioração. Com a remoção desse tipo de água, como verificado na secagem até $30 \%$, que levou o potencial de água para $-31,2 \mathrm{MPa}$, resta somente água dos tipos 1 e $2(<-30 \mathrm{MPa})$, que pode promover a morte das sementes recalcitrantes (Vertucci 1993).

A germinação inicial das sementes de $M$. pungens foi elevada (98\%, tabela 1), semelhante ao observado por outros autores (Santos et al. 2004, Fior et al. 2010), contudo mais rápida, como demonstrado pelo TMG (tabela 2). Apesar de poder ser considerada uma germinação rápida em relação a outras espécies arbóreas tropicais, também é uma germinação irregularmente distribuída no tempo, conforme observado pela VTM (tabela 3). Assim como também demonstrado nos trabalhos desses autores, confirma-se a intolerância à dessecação das sementes dessa espécie e, portanto, seu comportamento recalcitrante. As sementes não tiveram redução na germinação (tabela 1), nem na produção de plântulas normais (tabela 5) após secagem até $43 \%$, o que 
Tabela 1. Germinação de sementes de guabiju, Myrcianthes pungens (O. Berg) D. Legrand (Myrtaceae) com diferentes níveis de teor de água e armazenadas por até 66 dias em diferentes temperaturas, com e sem presença de luz. Médias seguidas pela mesma letra (minúsculas para comparação dentro das colunas, maiúsculas dentro das linhas), não diferem entre si pelo teste de Tukey (5\%).

Table 1. Germination of guabiju seeds, Myrcianthes pungens (O. Berg) D. Legrand (Myrtaceae) at different levels of water content and stored for up to 66 days at different temperatures, with or without light. Means followed by the same letter (lower case for comparison within columns, upper case within lines), do not differ by Tukey's test (5\%).

\begin{tabular}{cccrrrr}
\hline & Armazenamento & \multicolumn{5}{c}{ Teor de água } \\
\hline Tempo (dias) & Temperatura $\left({ }^{\circ} \mathrm{C}\right)$ & Luz & $50 \%$ & $43 \%$ & $30 \%$ \\
\hline 0 (inicial) & - & - & $98 \mathrm{Aa}$ & $100 \mathrm{Aa}$ & $23 \mathrm{Ba}$ \\
15 & 25 & presente & $100 \mathrm{Aa}$ & $97 \mathrm{Aa}$ & $5 \mathrm{Bcd}$ \\
15 & 25 & ausente & $100 \mathrm{Aa}$ & $93 \mathrm{Aa}$ & $10 \mathrm{Bbcd}$ \\
15 & 10 & presente & $98 \mathrm{Aa}$ & $97 \mathrm{Aa}$ & $2 \mathrm{Bcd}$ \\
15 & 10 & ausente & $98 \mathrm{Aa}$ & $97 \mathrm{Aa}$ & $3 \mathrm{Bcd}$ \\
30 & 25 & presente & $93 \mathrm{Aa}$ & $98 \mathrm{Aa}$ & $3 \mathrm{Bcd}$ \\
30 & 25 & ausente & $100 \mathrm{Aa}$ & $98 \mathrm{Aa}$ & $20 \mathrm{Bab}$ \\
30 & 10 & presente & $100 \mathrm{Aa}$ & $98 \mathrm{Aa}$ & 2 & $\mathrm{Bcd}$ \\
30 & 10 & ausente & $100 \mathrm{Aa}$ & $100 \mathrm{Aa}$ & 0 & $\mathrm{Bd}$ \\
66 & 25 & presente & - & $95 \mathrm{Aa}$ & $2 \mathrm{Bbc}$ \\
66 & 25 & ausente & - & $93 \mathrm{Aa}$ & $18 \mathrm{Bab}$ \\
66 & 10 & presente & $97 \mathrm{Aa}$ & $97 \mathrm{Aa}$ & $2 \mathrm{Bcd}$ \\
66 & 10 & ausente & $100 \mathrm{Aa}$ & $97 \mathrm{Aa}$ & $3 \mathrm{Bcd}$ \\
\hline
\end{tabular}

Tabela 2. Tempo médio para germinação de sementes de guabiju, Myrcianthes pungens (O. Berg) D. Legrand (Myrtaceae) com diferentes níveis de teor de água e armazenadas por até 66 dias em diferentes temperaturas, com e sem presença de luz. Médias seguidas pela mesma letra (minúsculas para comparação dentro das colunas, maiúsculas dentro das linhas), não diferem entre si pelo teste de Tukey (5\%).

Table 2. Mean germination time of guabiju seeds, Myrcianthes pungens (O. Berg) D. Legrand (Myrtaceae) at different levels of water content and stored for up to 66 days at different temperatures, with or without light. Means followed by the same letter (lower case for comparison within columns, upper case within lines), do not differ by Tukey’s test (5\%).

\begin{tabular}{cccrccc}
\hline & Armazenamento & & \multicolumn{5}{c}{ Teor de água } \\
\hline Tempo (dias) & Temperatura $\left({ }^{\circ} \mathrm{C}\right)$ & Luz & \multicolumn{1}{c}{$50 \%$} & $43 \%$ & $30 \%$ \\
\hline 0 (inicial) & - & - & $15,18 \mathrm{Aa}$ & $24,32 \mathrm{Aa}$ & - \\
15 & 25 & presente & $8,70 \mathrm{Aa}$ & $33,10 \mathrm{Ba}$ & - \\
15 & 25 & ausente & $8,10 \mathrm{Aa}$ & $30,18 \mathrm{Ba}$ & - \\
15 & 10 & presente & $13,81 \mathrm{Aa}$ & $32,78 \mathrm{Aa}$ & - \\
15 & 10 & ausente & $11,32 \mathrm{Aa}$ & $29,84 \mathrm{Aa}$ & - \\
30 & 25 & presente & $7,08 \mathrm{Aa}$ & $32,38 \mathrm{Ba}$ & - \\
30 & 25 & ausente & $6,95 \mathrm{Aa}$ & $47,39 \mathrm{Ba}$ & - \\
30 & 10 & presente & $12,4 \mathrm{Aa}$ & $30,95 \mathrm{Aa}$ & - \\
30 & 10 & ausente & $11,52 \mathrm{Aa}$ & $31,36 \mathrm{Ba}$ & - \\
66 & 25 & presente & - & $36,30 \mathrm{Aa}$ & - \\
66 & 25 & ausente & - & $32,30 \mathrm{Aa}$ & - \\
66 & 10 & presente & $10,30 \mathrm{Aa}$ & $42,39 \mathrm{Ba}$ & - \\
66 & 10 & ausente & $10,94 \mathrm{Aa}$ & $43,04 \mathrm{Ba}$ & - \\
\hline
\end{tabular}


Tabela 3. Variância do tempo médio de germinação de sementes de guabiju, Myrcianthes pungens (O. Berg) D. Legrand (Myrtaceae) com diferentes níveis de teor de água e armazenadas por até 66 dias em diferentes temperaturas, com e sem presença de luz. Médias seguidas pela mesma letra (minúsculas para comparação dentro das colunas, maiúsculas dentro das linhas), não diferem entre si pelo teste de Tukey (5\%).

Table 3. Variance of germination time of guabiju seeds, Myrcianthes pungens (O. Berg) D. Legrand (Myrtaceae) at different levels of water content and stored for up to 66 days at different temperatures, with or without light. Means followed by the same letter (lower case for comparison within columns, upper case within lines), do not differ by Tukey's test (5\%).

\begin{tabular}{ccccccc}
\hline & Armazenamento & & \multicolumn{5}{c}{ Teor de água } \\
\hline Tempo (dias) & Temperatura $\left({ }^{\circ} \mathrm{C}\right)$ & Luz & $50 \%$ & $43 \%$ & $30 \%$ \\
\hline 0 (inicial) & - & - & $85,43 \mathrm{Aa}$ & $112,82 \mathrm{Aa}$ & - \\
15 & 25 & presente & $49,54 \mathrm{Aa}$ & $152,32 \mathrm{Aa}$ & - \\
15 & 25 & ausente & $18,36 \mathrm{Aa}$ & $61,12 \mathrm{Aa}$ & - \\
15 & 10 & presente & $95.63 \mathrm{Aa}$ & $109,28 \mathrm{Aa}$ & - \\
15 & 10 & ausente & $22,22 \mathrm{Aa}$ & $127,49 \mathrm{Aa}$ & - \\
30 & 25 & presente & $9,60 \mathrm{Aa}$ & $102,46 \mathrm{Aa}$ & - \\
30 & 25 & ausente & $11,92 \mathrm{Aa}$ & $632,86 \mathrm{Bb}$ & - \\
30 & 10 & presente & $77,28 \mathrm{Aa}$ & $97,66 \mathrm{Aa}$ & - \\
30 & 10 & ausente & $40,41 \mathrm{Aa}$ & $85,30 \mathrm{Aa}$ & - \\
66 & 25 & presente & - & $347,64 \mathrm{Aa}$ & - \\
66 & 25 & ausente & & - & $162,46 \mathrm{Aa}$ & - \\
66 & 10 & presente & $28,33 \mathrm{Aa}$ & $159,81 \mathrm{Aa}$ & - \\
66 & 10 & ausente & $71,52 \mathrm{Aa}$ & $238,18 \mathrm{Aa}$ & - \\
\hline
\end{tabular}

poderia ser esperado uma vez que tais sementes podem ser dispersas com esse teor (Fior et al. 2010). Contudo, os valores de IVG (tabela 4) demonstraram que as sementes com $43 \%$ também tiveram queda no vigor, corroborando Vertucci (1993) e Marcos Filho (2005) de que a desidratação de sementes recalcitrantes até o nível 3 promove a deterioração destas de forma semelhante às ortodoxas (tolerantes à dessecação) submetidas a elevada umidade relativa do ar. A secagem até $30 \%$, por sua vez, resultou em redução já na porcentagem de germinação (tabela 1) e consequentemente na produção de plântulas normais (tabela 5), que era previsto, uma vez que estas sementes tiveram toda a água do tipo 3 removida, conforme mencionado anteriormente. Tal fato corrobora Wielewicki et al. (2006), que propuseram o padrão mínimo de $34,8 \%$ de água para sementes de $M$. pungens.

A intolerância à dessecação até $30 \%$ situaria essas sementes entre as mais sensíveis à dessecação (mais recalcitrantes), tal como as de Inga vera (Andréo et al. 2006, Bonjovani \& Barbedo 2014) e várias espécies de Eugenia (Delgado \& Barbedo 2007, Françoso \& Barbedo 2014), estas últimas pertencentes a Myrtaceae, mesma família da espécie deste trabalho. Contudo, diferentemente das sementes de muitas espécies que são altamente sensíveis à dessecação, as de $M$. pungens surpreendentemente conservaram-se bem em temperatura ambiente $\left(25^{\circ} \mathrm{C}\right)$ pelos primeiros 30 dias (tabela 1). Essas sementes estavam metabolicamente ativas, com elevadas taxas de respiração, como demonstrado pelo consumo de $\mathrm{O}_{2}$ e liberação de $\mathrm{CO}_{2}$ (figura 1), ou seja, não estavam em condição de conservação, mas de germinação muito lenta, provavelmente pela limitação de água. Os resultados de TMG (tabela 2), que foram menores após armazenamento a $25^{\circ} \mathrm{C}$, corroboram tal ideia. De fato, após 66 dias de armazenamento, $75 \%$ a $80 \%$ das sementes não submetidas à secagem e armazenadas a $25{ }^{\circ} \mathrm{C}$ (respectivamente, na luz e no escuro) germinaram ainda na própria embalagem, o que explica a ausência de dados na tabela 1. Além disso, a grande diferença entre o consumo de $\mathrm{O}_{2}$ e a liberação de $\mathrm{CO}_{2}$ (figura 1) indica processos oxidativos não diretamente relacionados com a respiração. Essa oxidação, em sementes de diversas espécies como Caesalpinia echinata Lam. (Lamarca \& Barbedo 2012), resulta em rápida deterioração das sementes no armazenamento (Santos \& Barbedo 2017).

A viabilidade das sementes (não submetidas a secagem) após armazenamento demonstrou não haver diferença entre armazenamento na luz e no escuro (tabela 1), diferentemente do observado para sementes de Salix nigra Marsh. (Roqueiro et al. 2010) que apresentaram 
Tabela 4. Índice de velocidade de germinação de sementes de guabiju, Myrcianthes pungens (O. Berg) D. Legrand (Myrtaceae) com diferentes níveis de teor de água e armazenadas por até 66 dias em diferentes temperaturas, com e sem presença de luz. Médias seguidas pela mesma letra (minúsculas para comparação dentro das colunas, maiúsculas dentro das linhas), não diferem entre si pelo teste de Tukey (5\%).

Table 4. Index of velocity of germination of guabiju seeds, Myrcianthes pungens (O. Berg) D. Legrand (Myrtaceae) at different levels of water content and stored for up to 66 days at different temperatures, with or without light. Means followed by the same letter (lower case for comparison within columns, upper case within lines), do not differ by Tukey's test (5\%).

\begin{tabular}{ccccccc}
\hline & Armazenamento & \multicolumn{5}{c}{ Teor de água } \\
\hline Tempo (dias) & Temperatura $\left({ }^{\circ} \mathrm{C}\right)$ & Luz & $50 \%$ & $43 \%$ & $30 \%$ \\
\hline 0 (inicial) & - & - & $1,22 \mathrm{Af}$ & $0,71 \mathrm{Ba}$ & $0,070 \mathrm{Ca}$ \\
15 & 25 & presente & $2,16 \mathrm{Aab}$ & $0,49 \mathrm{Bbc}$ & $0,016 \mathrm{Ca}$ \\
15 & 25 & ausente & $2,14 \mathrm{Ab}$ & $0,50 \mathrm{Bbc}$ & $0,033 \mathrm{Ca}$ \\
15 & 10 & presente & $1,43 \mathrm{Ae}$ & $0,46 \mathrm{Bbc}$ & $0,010 \mathrm{Ca}$ \\
15 & 10 & ausente & $1,51 \mathrm{Ade}$ & $0,53 \mathrm{Babc}$ & $0,010 \mathrm{Ca}$ \\
30 & 25 & presente & $2,16 \mathrm{Aab}$ & $0,50 \mathrm{Bbc}$ & $0,030 \mathrm{Ca}$ \\
30 & 25 & ausente & $2,36 \mathrm{Aa}$ & $0,59 \mathrm{Bab}$ & $0,080 \mathrm{Ca}$ \\
30 & 10 & presente & $1,68 \mathrm{Acd}$ & $0,52 \mathrm{Babc}$ & $0,009 \mathrm{Ca}$ \\
30 & 10 & ausente & $1,67 \mathrm{Acd}$ & $0,52 \mathrm{Babc}$ & $0,000 \mathrm{Ca}$ \\
66 & 25 & presente & - & $0,52 \mathrm{Aabc}$ & $0,032 \mathrm{Ba}$ \\
66 & 25 & ausente & - & $0,50 \mathrm{Abc}$ & $0,036 \mathrm{Ba}$ \\
66 & 10 & presente & $1,74 \mathrm{Ac}$ & $0,38 \mathrm{Bc}$ & $0,006 \mathrm{Ca}$ \\
66 & 10 & ausente & $1,84 \mathrm{Ac}$ & $0,38 \mathrm{Bc}$ & $0,012 \mathrm{Ca}$ \\
\hline
\end{tabular}

Tabela 5. Porcentagem de plântulas normais de sementes de guabiju, Myrcianthes pungens (O. Berg) D. Legrand (Myrtaceae) com diferentes níveis de teor de água e armazenadas por até 66 dias em diferentes temperaturas, com e sem presença de luz. Médias seguidas pela mesma letra (minúsculas para comparação dentro das colunas, maiúsculas dentro das linhas), não diferem entre si pelo teste de Tukey (5\%).

Table 5. Percentage of normal seedlings of guabiju seeds, Myrcianthes pungens (O. Berg) D. Legrand (Myrtaceae) at different levels of water content and stored for up to 66 days at different temperatures, with or without the presence of light. Means followed by the same letter (lower case for comparison within columns, upper case within lines), do not differ by Tukey's test (5\%).

\begin{tabular}{|c|c|c|c|c|c|c|c|c|}
\hline \multicolumn{3}{|c|}{ Armazenamento } & \multicolumn{6}{|c|}{ Teor de água } \\
\hline \multirow{2}{*}{$\begin{array}{c}\text { Tempo (dias) } \\
0 \text { (inicial) }\end{array}$} & \multirow{2}{*}{$\begin{array}{c}\text { Temperatura }\left({ }^{\circ} \mathrm{C}\right) \\
-\end{array}$} & \multirow{2}{*}{$\begin{array}{c}\text { Luz } \\
-\end{array}$} & \multicolumn{2}{|c|}{$50 \%$} & \multicolumn{2}{|c|}{$43 \%$} & \multicolumn{2}{|c|}{$30 \%$} \\
\hline & & & 78 & $\mathrm{Bab}$ & 97 & $\mathrm{Aa}$ & & $\mathrm{Ca}$ \\
\hline 15 & 25 & presente & 85 & $\mathrm{Aa}$ & 87 & Aabc & 3 & $\mathrm{Ba}$ \\
\hline 15 & 25 & ausente & 85 & $\mathrm{Aa}$ & 77 & Aabc & 5 & $\mathrm{Ba}$ \\
\hline 15 & 10 & presente & 63 & $\mathrm{Ab}$ & 72 & $\mathrm{Abc}$ & 0 & $\mathrm{Ba}$ \\
\hline 15 & 10 & ausente & 92 & $\mathrm{Aa}$ & 83 & Aabc & 3 & $\mathrm{Ba}$ \\
\hline 30 & 25 & presente & 77 & $\mathrm{Aab}$ & 88 & Aabc & 2 & $\mathrm{Ba}$ \\
\hline 30 & 25 & ausente & 87 & $\mathrm{Aa}$ & 82 & Aabc & 13 & $\mathrm{Ba}$ \\
\hline 30 & 10 & presente & 72 & $\mathrm{Bab}$ & 97 & $\mathrm{Aa}$ & 2 & $\mathrm{Ca}$ \\
\hline 30 & 10 & ausente & 80 & $\mathrm{Aab}$ & 90 & Aab & 0 & $\mathrm{Ca}$ \\
\hline 66 & 25 & presente & - & & 70 & $\mathrm{Abc}$ & 8 & $\mathrm{Ba}$ \\
\hline 66 & 25 & ausente & - & & 68 & Ac & 10 & $\mathrm{Ba}$ \\
\hline 66 & 10 & presente & 87 & $\mathrm{Aa}$ & 85 & Aabc & 0 & $\mathrm{Ba}$ \\
\hline 66 & 10 & ausente & 85 & $\mathrm{Aa}$ & 78 & Aabc & 0 & $\mathrm{Ba}$ \\
\hline
\end{tabular}


sensibilidade à foto-oxidação. Contudo, outros processos oxidativos devem ter ocorrido no armazenamento a $25^{\circ} \mathrm{C}$, pois na avaliação das trocas gasosas das sementes

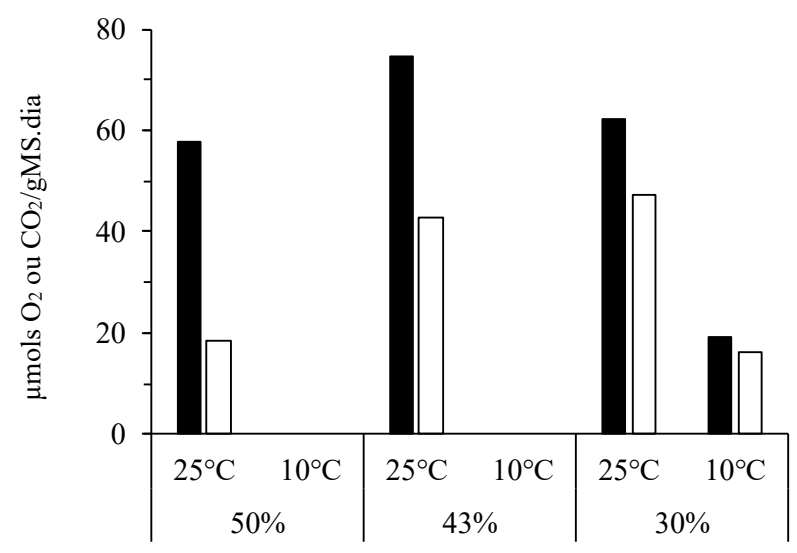

Temperatura de incubação e teor de água das sementes

Figura 1. Consumo de $\mathrm{O}_{2}$ (colunas pretas) e liberação de $\mathrm{CO}_{2}$ (colunas brancas) de sementes de guabiju, Myrcianthes pungens (O. Berg) D. Legrand (Myrtaceae) com diferentes níveis de teor de água e incubadas a $25^{\circ} \mathrm{C}$ e $10^{\circ} \mathrm{C}$.

Figure 1. Consumption of $\mathrm{O}_{2}$ (black columns) and release of $\mathrm{CO}_{2}$ (white columns) from seeds of guabiju, Myrcianthes pungens (O. Berg) D. Legrand (Myrtaceae) with different levels of water content and incubated at $25^{\circ} \mathrm{C}$ and $10{ }^{\circ} \mathrm{C}$. incubadas por 15 dias, como mencionado anteriormente, foi grande a diferença entre o $\mathrm{O}_{2}$ consumido e o $\mathrm{CO}_{2}$ liberado (figura 1). Em C. echinata Lam. (Lamarca \& Barbedo, 2012), essa diferença foi tanto maior quanto mais elevada a temperatura.

Quando submetidas à secagem até $43 \%$, as sementes armazenadas em todas as temperaturas e condições de luz apresentaram aumento no TMG (tabela 2). As não submetidas à secagem ( $50 \%$ de água) e armazenadas em temperatura baixa $\left(10^{\circ} \mathrm{C}\right)$, por sua vez, mantiveram tanto a elevada porcentagem inicial quanto a velocidade de germinação durante o período de armazenamento de até 66 dias.

As sementes com $50 \%$ de água armazenadas em baixa temperatura $\left(10^{\circ} \mathrm{C}\right)$ por 180 e 240 dias foram submetidas a testes de germinação com diferentes temperaturas, que variaram de $2{ }^{\circ} \mathrm{C}$ a $40{ }^{\circ} \mathrm{C}$. Os resultados mostram que nas temperaturas entre $15{ }^{\circ} \mathrm{C}$ e $25^{\circ} \mathrm{C}$, a porcentagem de germinação e a produção de plântulas normais mantiveramse elevadas (tabela 6). Em temperatura baixa, com baixo metabolismo e baixas taxas de oxidação (figura 1 e tabela 6), essas sementes provavelmente poderiam ser armazenadas por períodos maiores, como observado por Fior et al. (2010) após oito meses de armazenamento a $5^{\circ} \mathrm{C}$.

Assim, as sementes de Myrcianthes pungens podem ser consideradas como intolerantes à dessecação até valores de $30 \%$ de água. São melhores armazenadas sob temperaturas de $10{ }^{\circ} \mathrm{C}$ e, sob temperaturas mais elevadas, apresentam elevadas taxas tanto de respiração como de outros processos oxidativos.

Tabela 6. Porcentagem de germinação e de plântulas normais de sementes de guabiju, Myrcianthes pungens (O. Berg) D. Legrand (Myrtaceae) obtidas a partir de sementes armazenadas por 180 e 240 dias a $10^{\circ} \mathrm{C}$, sob diferentes temperaturas. Médias seguidas pela mesma letra não diferem entre si pelo teste de Tukey (5\%).

Table 6. Percentage of germination and normal seedlings of guabiju seeds, Myrcianthes pungens (O. Berg) D. Legrand (Myrtaceae) obtained from seeds stored for 180 and 240 days at $10{ }^{\circ} \mathrm{C}$, at different temperatures. Means followed by the same letter do not differ by Tukey's test (5\%).

\begin{tabular}{cccc}
\hline Tempo (dias) & Temperatura $\left({ }^{\circ} \mathrm{C}\right)$ & Germinação $(\%)$ & Plântulas (\%) \\
\hline 180 & 2 & $75 \mathrm{~b}$ & $0 \mathrm{~d}$ \\
180 & 5 & $95 \mathrm{a}$ & $0 \mathrm{~d}$ \\
180 & 10 & $97 \mathrm{a}$ & $30 \mathrm{c}$ \\
180 & 15 & $98 \mathrm{a}$ & $85 \mathrm{ab}$ \\
180 & 20 & $87 \mathrm{a}$ & $77 \mathrm{~b}$ \\
180 & 25 & $98 \mathrm{a}$ & $93 \mathrm{a}$ \\
180 & 30 & $13 \mathrm{c}$ & $5 \mathrm{~d}$ \\
180 & 35 & $8 \mathrm{c}$ & $0 \mathrm{~d}$ \\
180 & 40 & $0 \mathrm{c}$ & $0 \mathrm{~d}$ \\
240 & 25 & 83 & 50 \\
\hline
\end{tabular}




\section{Agradecimentos}

Os autores agradecem ao Sr. Albino De Bortoli, pelo fornecimento das sementes.

\section{Literatura citada}

Andrade, J.M.M., Aboy, A.L., Apel, M.A., Raseira, M.C.B., Pereira, J.F.M. \& Henriques, A.T. 2011. Phenolic composition in different genotypes of guabiju fruits (Myrcianthes pungens) and their potential as antioxidant and antichemotactic agents. Food Chemistry 76: 1181-1187.

Andréo, Y., Nakagawa, J. \& Barbedo, C.J. 2006. Mobilização de água e conservação da viabilidade de embriões de sementes recalcitrantes de ingá (Inga vera Willd. subsp. affinis (DC.) T. D. Pennington). Revista Brasileira de Botânica 29: 309-318.

Barbedo, C.J. 2018. A new approach towards the so-called recalcitrant seeds. Journal of Seed Science 40: 221-236.

Barbedo C.J., Centeno D.C., Figueiredo-Ribeiro R.C.L. 2013. Do recalcitrant seeds really exist? Hoehnea 40: 583-593.

Barbedo, C.J. \& Santos Junior, N.A. 2018. Sementes do Brasil: produção e tecnologia para espécies da flora brasileira. Instituto de Botânica, São Paulo.

Barbedo, C.J., Silva, J.P.N., Françoso, C.F. \& Parisi, J.J.D. 2018. Armazenamento de sementes. In Barbedo, C.J. \& Santos Junior, N.A. (orgs.) Sementes do Brasil: produção e tecnologia para espécies da flora brasileira. Instituto de Botânica, São Paulo. pp. 81-108.

Bonjovani, M.R. \& Barbedo, C.J. 2014. Induction of tolerance to desiccation and to subzero temperatures in embryos of recalcitrant seeds of inga. Journal of Seed Science 36: 419-426.

Borges, I.F., Giudice Neto, J.D., Bilia, D.A.C., FigueiredoRibeiro, R.C.L. \& Barbedo, C.J. 2005. Maturation of seeds of Caesalpinia echinata Lam. (brazilwood), an endangered leguminous tree from the Brazilian Atlantic Forest. Brazilian Archives of Biology and Technology 48: 851-861.

Borghetti, F. \& Ferreira, A.G. 2004. Interpretação de resultados de germinação. In Ferreira, A.G. \& Borghetti, F. (orgs.) Germinação: do básico ao aplicado. Artmed, Porto Alegre. pp. 209-222.

Brasil. 2009. Regras para análise de sementes. Ministério da Agricultura, Pecuária e Abastecimento, Secretaria de Defesa Agropecuária, Coordenação Geral de Apoio Laboratorial, Brasília.

Delgado, L.F. \& Barbedo, C.J. 2007. Tolerância à dessecação de sementes de espécies de Eugenia. Pesquisa Agropecuária Brasileira 42: 265-272.
Fior, C.S., Rodrigues, L.R., Calil, A.C., Leonhardt, C., Souza, L.S. \& Silva, V.S. 2010. Qualidade fisiológica de sementes de guabijuzeiro (Myrcianthes pungens (Berg) Legrand - Myrtaceae) em armazenamento. Revista Árvore 34: 435-442.

Françoso, C.F. \& Barbedo, C.J. 2014. Tratamentos osmóticos e térmicos para controle de fungos em sementes de grumixameira (Eugenia brasiliensis Lam.) e pitangueira (Eugenia uniflora L.). Hoehnea 41: 541-552.

Guardia, M.C., Asperti, L.M., Fidalgo, A.O. \& Garcia, V.A. 2018. Produção e obtenção de sementes. In Barbedo, C.J. \& Santos Junior, N.A. (orgs.) Sementes do Brasil: produção e tecnologia para espécies da flora brasileira. Instituto de Botânica, São Paulo. pp. 41-62.

Gibbert, P., Malavasi, M.M., Malavasi, U.C.\& Dranski, J.A.L. 2019. Conservation of seeds of Myrcianthes pungens (BErg.) Legr. in different packaging in a controlled environment. Revista Árvore 43: e430205.

Kader, A.A. \& Saltveit, M.E. 2002. Respiration and gas exchange. In Bartz, J.A., Brecht, J.K. \& Weichmann, J. (orgs.) Postharvest physiology and pathology of vegetables. Marcel Deckker, New York. pp. 7-29.

Lamarca, E.V. \& Barbedo, C.J. 2012. Short storability of Caesalpinia echinata Lam. seeds as a consequence of oxidative processes. Hoehnea 39: 577-586.

Lamarca, E.V., Baptista, W., Rodrigues, D.S \& Oliveira Júnior, C.J.F. 2013a. Contribuições do conhecimento local sobre o uso de Eugenia spp. em sistemas de policultivos e agroflorestas. Revista Brasileira de Agroecologia 8: 119-130.

Lamarca, E.V., Prataviera, J.S., Borges, I.F., Delgado, L.F., Teixeira, C.C., Camargo, M.B.P., Faria, J.M.R., Barbedo, C.J. 2013b. Maturation of Eugenia pyriformis seeds under different hydric and thermal conditions. Anais da Academia Brasileira de Ciencias 85: 223-233.

Lamarca, E.V., Camargo, M.B.P., Teixeira, S.P., Silva, E.A.A., Faria, J.M.R. \& Barbedo, C.J. 2016. Variations in desiccation tolerance in seeds of Eugenia pyriformis: dispersal at different stages of maturation. Revista Ciencia Agronomica 47: 118-126.

Lamont, B.B., He, T. \& Yan, Z. 2019. Evolutionary history on fire-stimulated resprouting, flowering, seed release and germination. Biological Reviews 94: 903-928.

Linkies, A., Graeber, K., Knight, C., Leubner, G. 2010. The evolution of seeds. New Phytologist 186: 817-831.

Magnani, E. 2018. Seed evolution, a 'simpler' story. Trends in Plant Science 23: 654-656.

Marcos Filho, J. 2015. Fisiologia de sementes de plantas cultivadas. Abrates, Londrina. 
Nora, C.D., Jablonski, A., Rios, A.O., Hertz, P.F., Jong, E.V. \& Flôres, S.H. 2014. The characterisation and profile of the bioactive compounds in red guava (Psidium cattleyanum Sabine) and guabiju (Myrcianthes pungens (O. Berg) D. Legrand). International Journal of Food Science and Technology 49: 1842-1849.

Roqueiro, G., Facorro, G.B., Huarte, M.G., Celis, E.R., García, F., Maldonado, S. \& Maroder, H. 2010. Effects of photooxidation on membrane integrity in Salix nigra seeds. Annals os Botany 105: 1027-1034.

Sano, S.M., Vivaldi, L.J. \& Spehar, C.R. 1999. Diversidade morfológica de frutos e sementes de baru (Dipteryx alata Vog.). Pesquisa Agropecuária Brasileira 34: 513-518.

Santana, D.G. \& Ranal, M. 2004. Análise da germinação: um enfoque estatístico. Ed. Universidade de Brasília, Brasília.

Santos, M.R.) \& Barbedo, C.J. 2017. Taxas de deterioração de sementes de pau-brasil (Caesalpinia echinata Lam.) sob elevadas temperaturas. Hoehnea 44: 449-463.

Santos, C.M.R., Ferreira, A.G. \& Áquila, M.E.A. 2004. Características de frutos e germinação de sementes de seis espécies de Myrtaceae nativas do Rio Grande do Sul. Ciência Florestal 14: 13-20.

Silva, M.R. \& Lamarca, E.V. 2018. Registros etnobotânicos e potenciais medicinais e econômicos do jatobá (Hymenaea courbaril). Revista da Universidade Ibirapuera 15: 8-12.
Silva, E.A.A., Oliveira, J.M. \& Pereira, W.V.S. 2018. Fisiologia das sementes. In Barbedo, C.J. \& Santos Junior, N.A. (orgs.) Sementes do Brasil: produção e tecnologia para espécies da flora brasileira. Instituto de Botânica, São Paulo. pp. 15-40.

Subbiah, A., Ramdhani, S., Pammenter, N.W., Macdonald, A.H.H. \& Sershen. 2019. Towards understanding the incidence and evolutionary history of seed recalcitrance: an analytical review. Perspectives in Plant Ecology, Evolutions and Systematics 37: 11-19.

Vertucci, C.W. 1993. Predicting the optimum storage conditions for seeds using thermodynamic principles. Journal of Seed Technology 17: 41-53.

Wielewicki, A.P., Leonhardt, C., Schlindwein, G. \& Medeiros, A.C.S. 2006. Proposta de padrões de germinação e teor de água para sementes de algumas espécies florestais presentes na região Sul do Brasil. Revista Brasileira de Sementes 28: 191-197.

Wu, H., Wang, S., Wei, X. \& Jiang, M. 2019. Sensitivity of seed germination to temperature of a relict tree species from different origins along latitudinal and altitudinal gradients: implications for response to climate change. Trees, Structure and Function 33: 1435-1445. 